\title{
Les représentations des Autochtones dans les manuels scolaires et le respect de la Convention relative aux droits de l'enfant.
}

\section{Joannie St-Pierre}

Faculté d'éducation, étudiante au doctorat, Université d'Ottawa

\begin{abstract}
Résumé
Les manuels scolaires sont des artéfacts culturels illustrant des valeurs, des idéologies et des croyances. Afin d'en approfondir le contenu latent, une analyse critique du discours a été effectuée au sein de neuf manuels qui étaient recommandés pour introduire les notions relatives à la citoyenneté en cinquième et sixième année dans les écoles de langue française de l'Ontario. Les données ont été questionnées sous trois angles : la structure et les caractéristiques, la construction des personnages et les omissions. La recherche révèle que le discours portant sur les représentations des Autochtones ne respecte pas

l'article 28, l'article 29 et l'article 30 de la Convention relative aux droits de l'enfant. Les suggestions qui découlent de cette recherche devraient affiner l'évaluation des futures publications afin que les représentations des communautés autochtones au sein du matériel scolaire soient plus justes.

Mots-clés : Autochtone ; droit de l'enfant ; représentation ; manuel scolaire ; contenu latent.
\end{abstract}

\begin{abstract}
School textbooks are cultural artefacts that illustrate values, ideologies and beliefs. In order to expand upon the latent content, a critical discourse analysis was conducted on nine textbooks which were recommended to introduce the notion of citizenship to students in fifth and sixth grade in French language schools in Ontario. This data was examined from three angles: the structure and characteristics, the construction of the characters, and the omissions. This research reveals that Articles 28, 29 and 30 of the Convention on the Rights of the Child were not respected with regard to the representation of Indigenous peoples. The suggestions that result from this research should provide further improvement of the evaluation criteria for future publications, ensuring a better representation of Aboriginal communities.
\end{abstract}

Key words: Aboriginal, Indigenous, rights of the child, representation, school textbook, latent content. 


\section{Introduction}

Selon Morand (2014), 40 \% de la population autochtone de l'Ontario âgée de plus de 15 ans n'a pas obtenu un diplôme d'études secondaires. Devant cette statistique troublante et conformément au droit de l'enfant à l'éducation, le ministère de l'Éducation de l'Ontario s'est doté de mesures pour accompagner les élèves autochtones et ainsi « encourager la régularité de la fréquentation scolaire et la réduction d'abandon scolaire » (Convention relative aux droits de l'enfant, 1989, article 28). Depuis 2007, une série d'initiatives ont été mises en place dont une politique d'auto-identification volontaire et confidentielle pour les élèves autochtones. Celle-ci répond aux besoins des Conseils scolaires qui jadis étaient dans l'ignorance concernant le nombre d'élèves autochtones qui fréquentaient les écoles financées par la province. Cette politique a donc facilité l'accès à l'information dans le but d'orienter les décisions et les programmes. Bien que ces chiffres relatifs au nombre d'élèves autochtones soient confidentiels, Statistique Canada présente un portrait d'ensemble de la population autochtone ontarienne qui laisse croire qu'une certaine proportion des enfants autochtones de l'Ontario fréquentent les écoles de langue française. Entre autres, en 2006, 188310 Ontariens s'identifiaient comme autochtones. De ce nombre, 13560 s'identifiaient comme locuteurs du français uniquement, 65 comme locuteurs du français et de langue(s) autochtone(s) et 55 comme locuteurs du français, de langue(s) autochtone(s) et de l'anglais (Statistique Canada, 2006). Les enfants autochtones ont donc la possibilité de fréquenter une des 343 écoles de langue française de la province (MÉO, 2013). Il est donc pertinent d'interroger le discours présent dans ces institutions éducatives à propos de la réalité autochtone. Ainsi, afin de porter un regard critique sur le sujet, les représentations des Autochtones au sein du matériel éducatif ont été analysées. Après un survol des documents ministériels qui mettent en contexte l'approche préconisée par rapport à la diversité, une analyse critique du discours des manuels d'études sociales destinés aux élèves de cinquième et de sixième année fréquentant les écoles de langue française de l'Ontario est effectuée. Finalement, cette étude révèle qu'il y a un non-respect des droits préconisés par la Convention relative aux droits de l'enfant dans le contenu latent des manuels scolaires.

\section{La Convention relative aux droits de l'enfant et le discours portant sur la} diversité dans les documents ministériels.

Selon le projet de loi 13, l'éducation des enfants devrait être équitable et inclusive (Loi pour des écoles tolérantes, qui modifie la Loi sur l'éducation, entrée en vigueur le 1er septembre 2012). Cette loi prévoit que « tous les conseils scolaires devront 
offrir des milieux d'apprentissage sécuritaires, inclusifs et tolérants qui permettent à tous les élèves de réussir » (Assemblée législative de l'Ontario, 2012). Conséquemment à ces législations, le discours relatif aux droits de l'enfant dans les documents ministériels spécifiques aux écoles de langue française de l'Ontario s'ancre dans une conception inclusive. D'ailleurs, l'approche culturelle, proposée en 2009, cible l'appropriation de la culture francophone et vise la réussite scolaire et la construction identitaire des élèves fréquentant les écoles de langue française de l'Ontario. Plus spécifiquement, ce guide d'orientation et d'intervention suit les principes de la Convention relative aux droits de l'enfant quant au respect de son identité, de sa langue et ses valeurs culturelles. Quant aux identités ainsi qu'aux pratiques sociales et langagières multiples, le discours de ce document officiel est de l'ordre de l'ouverture, du respect, de la fierté, du sens d'appartenance, de la solidarité et de l'engagement (MÉO, 2009, p. 16). Cette approche favorise donc «l'épanouissement de la personnalité de l'enfant et le développement de ses dons et de ses aptitudes mentales et physiques, dans toute la mesure de leurs potentialités » (Article 29) en lui conférant le droit de construire son identité dans une culture flexible et renouvelée.

En ce qui concerne les élèves autochtones, les écoles de langue française de l'Ontario occupent différents rôles auprès de ceux-ci. Premièrement, comme mentionné précédemment, elles sont un milieu d'apprentissage qui vise la réussite de tous (MÉO, 2004). L'article 28 cadre avec ce mandat puisqu'il préconise la mise en place de mesures encourageant la réussite des élèves. Deuxièmement, comme exposé ci-haut, les écoles sont aussi un milieu de construction identitaire qui favorise le développement de l'identité culturelle et du sentiment d'appartenance à la culture franco-ontarienne (ibid.).

Conséquemment, si les écoles ont le mandat de promouvoir l'inclusion, l'article 29 est une balise incontournable pour favoriser l'épanouissement des enfants autochtones et pour inculquer le respect de leur identité, de leur langue et de leurs valeurs culturelles. De plus, en cohérence avec l'article 30 de la Convention, les principes préconisés par l'approche culturelle devraient permettre de reconnaitre le droit des enfants d'origine autochtone d'avoir leur propre vie culturelle et d'employer leur propre langue en commun avec les autres membres de leur groupe.

Le discours relatif aux droits de l'enfant dans le curriculum d'études sociales, d'histoire et de géographie

L'enseignement au respect des droits de l'homme et des libertés fondamentales (Article 29) est inculqué à l'enfant à travers le curriculum des études sociales, d'histoire et de géographie. Ainsi, suivant les principes consacrés dans la Convention relative aux droits de l'enfant, «l'enfant apprend le respect des valeurs nationales du pays dans lequel il vit, 
du pays duquel il peut être originaire et des civilisations différentes de la sienne » (ibid.). Toujours selon la Convention, l'enfant doit être préparé à assumer des responsabilités sociétales sous un regard compréhensif. Cet enseignement repose sur la paix, la tolérance, l'égalité entre les sexes et l'" amitié entre tous les peuples et groupes ethniques, nationaux et religieux, et avec les personnes d'origine autochtone » (ibid.). Dans le programme, il est question d'examiner des questions liées aux droits et aux responsabilités de la personne et de la société (MÉO, 2013, p. 12) afin d'« aider l'élève à devenir une citoyenne ou un citoyen responsable et actif au sein de ses groupes et communautés d'appartenance » (ibid., p. 8) dans une société inclusive.

Finalement, en concordance avec la Convention, le discours officiel préconise que l'élève soit amené à comprendre et à apprécier la diversité selon le principe de valorisation de l'inclusion. Le discours ministériel et le curriculum d'études sociales, d'histoire et de géographie demandent à ce que les ressources pédagogiques reflètent le pluralisme de la culture francophone dans toute sa diversité. Effectivement, puisque le matériel scolaire contribue à la construction identitaire des élèves (MÉO, 2009), le matériel d'apprentissage doit refléter la diversité de la communauté francophone et la société ontarienne dans son ensemble (MÉO, 2013). Mais est-ce bien le cas ?

\section{Les représentations de l'«Autre» dans les manuels scolaires}

D'abord, qu'est-ce que la littérature scientifique dit à propos des représentations de la diversité dans les manuels scolaires? Autrement dit, comment la diversité culturelle et identitaire y est-elle représentée? En Amérique du Nord, des recherches américaines, canadiennes et ontariennes s'attardent à ce sujet.

Du côté américain, des recherches ont démontré que le matériel scolaire représentant les diversités culturelles a un impact positif sur les résultats scolaires et sur l'estime de soi des élèves de ces communautés (Diamond et Moore, 1995). Pourtant, les représentations des membres et des groupes ethniques dans les manuels américains sont critiquées. Déjà, en 1991, Sleeter et Grant analysaient 47 manuels scolaires utilisés de la première à la huitième année et soulignaient qu'il y a non seulement peu de personnages représentant la diversité, mais qu'ils sont présentés sans complexité. Cette même année, McCarthy (1991) suggère aussi une réflexion dans ce sens suite à la recension des écrits des auteurs visant à rendre le curriculum américain plus multiculturel. Il en critique l'approche fragmentaire, soit la tendance à réduire un groupe à un personnage notoire. Il relève aussi l'omission de point de vue de groupes minoritaires. Puis, en 1993, DennickBrecht a analysé le contenu de trente manuels américains d'introduction à la sociologie. Il relève que seulement $6 \%$ du contenu est consacré au sujet de la diversité. Il associe donc 
cette sous-représentation aux fondements Eurocentriques de la sociologie. Finalement, de façon globale, les auteurs tels que Loewen (1995), Moreau (2003) ou encore Zimmerman (2002) soulignent la faible diversité dans les représentations de groupes ethniques (cités dans Hugues, 2007). Bref, les omissions, les sous-représentations et la construction des personnages font l'objet de la critique américaine.

Au Canada, la politique multiculturelle laisse anticiper une situation différente. D’ailleurs, dans les années quatre-vingt, McAndrew (1987) ne relevait ni préjugés ethniques ni racisme dans le contenu manifeste de 193 manuels scolaires québécois autorisés par le ministère de l'Éducation. Selon elle, le traitement du racisme, de l'immigration et de la réalité multiethnique y est complexe et non grossièrement stéréotypé. Par contre, sa recherche quantitative et qualitative relève un biais ethnocentrique constant privilégiant les préoccupations et visions du groupe majoritaire. Toutefois, dans les années quatre-vingt-dix, Blondin (1990) prend un autre chemin. Plutôt que de porter attention au contenu manifeste comme l'avait fait McAndrew, il approfondit le contenu latent des manuels, soit les théories et idéologies sous-jacentes au discours et transmises par celui-ci. Cette nouvelle approche révèle à l'auteur des fondements racistes découlant des catégorisations présentes dans les manuels scolaires. Selon lui, puisque les différenciations qu'il relève se basent sur des traits biologiques héréditaires et généralisés, la culture blanche domine la différenciation entre les groupes. Du côté albertain, Tupper (2002) étudie les représentations des Canadiens d'origine japonaise. Elle analyse trois manuels scolaires d'histoire utilisés en Alberta et dénonce le peu d'informations à propos des membres de ce groupe. En s'attardant aux présentations visuelles et au langage associé à cette communauté, elle relève plusieurs omissions quant au rôle et aux attitudes des Canadiens d'origine japonaise et quant à la discrimination dont ils ont été victimes. Sur cette même lancée, il n'est pas surprenant que Ghosh (2008) suggère de prêter attention au contenu latent des manuels scolaires.

Racist and sexist materials have largely been removed from formal curricula, although the problem of Eurocentric educational systems across Canada does produce racist effects, largely through textbooks non-recognition and misrecognition of the contribution of groups of peoples. (p. 4)

Finalement, Peck, Sears et Donalson (2008) confirme dans leur étude l'impact qu'a le message transmis dans les manuels scolaires sur les représentations de la citoyenneté canadienne des élèves nouveaux-brunswickois. Leur étude démontre que les élèves ont des connaissances très limitées du multiculturalisme et les auteurs recommandent que le matériel éducatif soit plus représentatif de la population scolaire. En d'autres mots, comme les recherches américaines, les recherches canadiennes révèlent des biais quant aux représentations des groupes ethniques. 
Plus spécifiquement, qu'en est-il en contexte scolaire ontarien ? D'abord, Montgomery (2005) a analysé 27 manuels scolaires d'histoire de $10^{\mathrm{e}}$ année. Son analyse de discours révèle que les traitements discriminatoires destinés aux Autochtones, aux Japonais pendant la $2^{\mathrm{e}}$ guerre mondiale ou aux immigrants sont présentés comme étant exceptionnels. Le racisme est justifié, dénié ou banalisé par l'attribution du tort aux individus ou à des événements isolés. Selon l'auteur, l'inclusion ou l'exclusion de certaines perspectives se trouve au sein même du processus de production des manuels scolaires par le simple fait de choisir d'ordonner, d'interpréter, de mettre en évidence ou d'omettre un événement ou une personne historique. Il souligne donc que l'histoire nationale repose sur un nationalisme ethnique en termes de consanguinité inspiré d'une pensée raciale (2005b). Il conclut que le choix du contenu, le déterminisme et la définition de la population, soit son inclusion ou son exclusion, restent basés sur la « racialisation ». Puis, Razai-Rashti et McCarthy (2008) ont examiné le contenu des manuels de langue anglaise des sciences sociales de 1'Ontario en 2002. Ils notent que 1'appartenance raciale et les différences sociales sont représentées dans les manuels de $12^{\mathrm{e}}$ année, mais que les discussions controversées sont absentes ou simplifiées. De plus, les actes discriminatoires réfèrent au passé ou sont associés à l'incompréhension. Ils en concluent qu'il y a présence d'une discrimination institutionnelle puisque les points de vue des groupes marginalisés sont ignorés ou biaisés.

\section{Les représentations des Autochtones dans les manuels scolaires}

Outre les critiques concernant l'inclusion de la diversité relative à divers groupes issus de l'immigration, la discrimination institutionnelle est aussi présente dans les représentations des groupes autochtones. Même si, en tant que peuple originel, leur apport historique ainsi que l'espace qui est consacré à leur vision du monde devrait transparaître dans les manuels scolaires, divers chercheurs américains et canadiens ont démontré que les Autochtones sont représentés de façons biaisées et qu'ils sont souvent ignorés. Entre autres, aux États-Unis, alors que Fleming (2006) s'attarde à déconstruire les stéréotypes et les mythes associés à la construction des personnages autochtones, Sanchez (2007) déplore que seulement trois manuels sur les quinze qu'il a analysés présentent de façon acceptable les Autochtones. Les douze autres compteraient des généralisations sans explications, des omissions et des inexactitudes. Du côté canadien, déjà en 1978, Vincent et Arcand (1978) abordent ces représentations sous l'angle de l'ethnocentrisme : «Les Amérindiens sont là lorsqu'ils servent de référence à l'histoire des Blancs, lorsqu'ils contribuent, finalement, à justifier l'identité spécifique des ex-Français » (cité dans Trudel, p. 532). Puis, en 1999, Bouchard note aussi les omissions des Premières Nations dans le contenu portant sur la 
période de la formation de l'État canadien. Selon lui, ils sont représentés lorsqu'il est question de revendications territoriales, mais l'accent demeure sur les peuples fondateurs et sur les autochtones du Nord. Finalement, en 2000, Trudel étudie la place qui est faite aux Autochtones dans les manuels scolaires dans une importante recension. Ceux-ci sont parfois représentés tels des participants à la nation, des victimes, des revendicateurs ou des communautés extérieures et distinctes.

Ainsi, inspiré de ces lectures, il est apparu nécessaire d'aborder la question des représentations des Autochtones dans le contenu latent du matériel scolaire afin d'approfondir la question du respect des droits des enfants autochtones.

\section{Méthodologie}

\section{Contexte}

Premièrement, pourquoi choisir le contexte minoritaire franco-ontarien ? Nasser et Nasser (2008) soulignent qu'en contexte minoritaire, le discours est nécessaire à la compréhension du processus de régulations des relations ethniques. De plus, ce discours est crucial à la formation de l'identité dans les sociétés ethniquement divisées. Leur affirmation concernant le rôle des écoles dans la promotion à l'intégration politique, économique et culturelle par rapport à la ségrégation des groupes dans la société est éclairante quant à la construction identitaire d'une personne (ibid., p. 629). Bien que ce contexte soit particulièrement intéressant, il est a noté que le contexte franco-ontarien est choisi pour sa grande diversité culturelle. Aucune supposition quant à l'attitude du milieu par rapport à la diversité ne justifie ce choix. De plus, d'autres écoles en contexte minoritaire canadien utilisent ces manuels. Aussi, à l'exception de deux manuels ayant été conçus pour répondre aux besoins éducatifs des écoles de langue française de l'Ontario, ces manuels sont le résultat de traduction. Ils ont donc une équivalence qui est proposée aux élèves anglophones.

\section{Choix de l'échantillon}

Deuxièmement, pourquoi choisir les manuels scolaires? Selon Crawford (2004), les manuels scolaires sont des artéfacts culturels qui reproduisent la société selon le point de vue du groupe dominant. Effectivement, les choix de contenu illustrent les valeurs et les idéologies importantes des groupes qui les approuvent. Le choix de la cinquième et de la sixième année est justifié par le fait que

the manner in which an author chooses to represent ethnic group members has great influence on the reader, especially in the elementary school years. It is therefore important to examine representations of ethnicities in textbooks to 
determine if there are any explicit or implicit negative messages created by the writer. (Mc Cabe, 1996, p. 381)

De plus, puisque les notions relatives à l'éducation à la citoyenneté sont introduites en cinquième et en sixième année (McAndrew, 2006), l'échantillon est constitué des manuels scolaires couvrant ces niveaux scolaires.

Plus spécifiquement, l'échantillon est formé de manuels scolaires ${ }^{\mathrm{i}}$ publiés par les maisons d'éditions La Chenelière et McGrawHill \& Oxford et faisant partie de la liste Trillium. Cette liste regroupe les manuels approuvés par le ministère de l'Éducation de l'Ontario. Pour se retrouver sur cette liste, un manuel doit préalablement être évalué par le Centre canadien de leadership en éducation (CLÉ). Certains critères de la CLÉ touchent à la non-discrimination, au non-jugement et à la non-exclusion. Le ministère s'attend à ce que tout préjugé fondé sur la race ou sur l'origine ethnoculturelle en soit exempt. De plus, il demande l'exemption de tout préjugé résultant d'une omission (MÉO, 2008).

\section{Objectif et questions de recherche}

À la base, cette étude analyse les représentations de l'« Autre ». Toutefois, dans le cadre de cet article, seules les informations relatives aux Autochtones sont présentées. Les deux questions de recherche sont les suivantes :

- Comment sont représentés les Autochtones dans les manuels scolaires d'études sociales destinés aux élèves de cinquième et de sixième année fréquentant les écoles de langue française de l'Ontario?

- Est-ce que ces représentations respectent les droits de l'enfant? Si non, quels droits sont non respectés?

Dans l'ensemble, l'objectif de cette recherche est de circonscrire les représentations des Autochtones dans le matériel scolaire en contexte francophone minoritaire. Cette étude est pertinente puisqu'il est à noter que depuis le 31 août 2015, aucun manuel n'est recommandé en Ontario pour couvrir cette matière et ces niveaux scolaires. Ainsi, les suggestions qui découlent de cette recherche devraient affiner l'évaluation des futures publications afin que les représentations des Autochtones au sein du matériel scolaire soient plus justes. De plus, les résultats pourraient sensibiliser les autres provinces utilisant ces manuels. Ultimement, des représentations plus justes permettraient le respect des droits des enfants autochtones tel que préconisé par les documents ministériels. 


\section{La méthode}

Au Canada, depuis les années soixante-dix, diverses approches ont tenté de faire place à la diversité. D'abord, selon un modèle anglo-saxon du multiculturalisme, l'individu peut appartenir à une communauté autre que l'État-nation. La société est présentée telle une mosaïque, mais l'accent sur les connaissances culturelles peut y agir tel un filtre. L'approche interculturelle cible davantage la compréhension de l'« Autre» et considère les connaissances culturelles telles des données construites. Ces deux approches ciblent l'harmonie entre les groupes, l'enrichissement mutuel et la valorisation de l'héritage culturel. L'approche antiraciste critique le multiculturalisme et l'approche interculturelle et leur reproche leur tendance à réduire les préjugés par la connaissance et la compréhension de l' «Autre ». L'approche antiraciste suggère plutôt que le racisme serait au cœur même des pratiques et des institutions des sociétés occidentales (McAndrew, 2006). Suivant cette dernière approche, l'étude présente tente de lever le voile sur les attitudes, les valeurs et les croyances présentes dans les relations entre le texte, le langage, les groupes sociaux et les pratiques sociales. L'analyse critique du discours guide la collecte et l'analyse de données puisque « [i]t tries to illuminate ways in which the dominant forces in a society construct versions of reality that favour their interests » (McGregor, 2003, p. 2). Dans un positionnement interprétatif, cette méthode permet d'approfondir les relations de pouvoirs présentes dans le discours.

\section{La collecte et l'analyse de données}

D'abord, le discours est abordé de façon non critique (McGregor, 2003). À la façon de Montgomery (2005), les façons d'illustrer, de nommer, de relier, de combiner, de signifier et d'ordonner le contenu dans les descriptions et dans les différenciations et catégorisations des groupes Autochtones sont identifiées à l'aide de vignettes. Puis, ces données ainsi que les commentaires sont compilés et révisés sous un angle critique. Les questionnements soulevés, inspirés de English learning area, interrogent a) la structure et les caractéristiques : soit par l'organisation des idées transmises, et les caractéristiques, soit par la distinction et les particularités d'un texte, soit par le choix des images, du vocabulaire ainsi que du langage ; b) la construction des personnages : soit les rôles joués par les gens, soit les personnages représentant certains groupes, soit les contextes et valeurs associées à des communautés ; c) les omissions : soit la présence et l'absence d'éléments qui peuvent manifester blocages, refoulements et volonté cachée (Bardin, 1989, p. 140); d) le pouvoir et les intérêts. Dans le cadre de cet article, seuls les trois premiers critères seront considérés. 


\section{Résultats : Les représentations des Autochtones dans les manuels d'études sociales de cinquième et de sixième année des écoles de langue française de l'Ontario}

\section{Structure et caractéristiques}

Au fil de l'analyse, les choix syntaxiques, du vocabulaire et des conjugaisons laissent sous-entendre que les inégalités semblent être chose ancienne ou externe. Effectivement, un premier pan des résultats concerne les extraits ayant des verbes conjugués au passé. Ces choix laissent à penser que la discrimination est chose du passé ou chose révolue. Par exemple, le fascicule Question de choix explique que les Autochtones ont jadis été privés de leurs droits. Puis, le discours de ce manuel propose qu'il y ait eu des « changements et qu'ils sont maintenant protégés par la Charte canadienne des droits et libertés » (Question de choix, 1999, p. 39). Donc, le discours au sein de l'échantillon de recherche omet de traiter certains débats et luttes actuels. Ainsi, l'idéologie véhiculée est celle d'un peuple vivant aujourd'hui en ayant les mêmes droits citoyens, mais certaines inégalités ou restrictions actuelles ne sont pas énoncées en ce qui concerne, par exemple, les droits à l'éducation dans une langue autochtone, la vie sur la réserve ou encore les droits territoriaux.

Un autre exemple portant sur les droits territoriaux dans le manuel Exploration (1999) est non seulement traité au passé dû à l'utilisation de l'imparfait lorsque le texte réfère aux Autochtones comparativement au temps présent pour les non-Autochtones, mais il y a aussi association entre l'idée de possession et les propriétaires de descendances autres qu'autochtones comparativement à l'idée d'habitation et les Autochtones :

Deux groupes se disputent ce territoire : les propriétaires des terrains autour du lac, qui possèdent des maisons à cet endroit depuis plusieurs années; les

Autochtones qui habitaient la région depuis des générations. (p. 40-14)

Cet extrait relevant des divergences de points de vue ne rappelle-t-il pas la nonreconnaissance des droits territoriaux des Autochtones?

À ces conjugaisons passées s'ajoute un second pan inspirant une certaine déresponsabilisation. En effet, il semble que lorsqu'il est question d'injustices, la responsabilité ne soit pas attribuée aux Canadiens de descendance européenne. Un premier exemple concerne les injustices vécues par les Métis, les Pieds-Noirs, les Cris et les Assiniboines. Celles-ci sont expliquées comme étant causées par le fait que «[1] e gouvernement canadien n'a pas tenu toutes ses promesses et beaucoup d'Autochtones luttent encore aujourd'hui pour obtenir un règlement plus juste » (Laisse sa marque, 1999, 
p. 37). Ainsi, plutôt que d'être associée au peuple, la responsabilité de l'injustice repose sur un ensemble politique. Aussi, un second exemple relève du choix de structurer certaines phrases sous la forme passive. Ce faisant, cela contribue à cette dépersonnalisation. Par exemple, l'auteur de Je découvre les Premières Nations et les explorateurs (2002) mentionne que «Les peuples autochtones ont été forcés d'abandonner leurs terres et ont été repoussés en marge de la société » (p. 26). Dans un autre extrait, cette dépersonnalisation face à des événements négatifs est présente sous couvert de l'utilisation du mode impersonnel « on »:

À mesure que les gens arrivaient d'Europe de plus en plus nombreux pour explorer le pays et s'y installer, les bisons ont commencé à avoir moins d'espace. On en a tué énormément. On a envoyé d'immenses quantités d'os de bisons aux États-Unis, où on les a transformés en engrais. (Je decouvre le Canada, 2002, p. 63)

Qui sont les «on»? Cette dépersonnalisation n'entraine-t-elle pas une forme de déresponsabilisation?

Toujours dans le questionnement de la structure et des caractéristiques du texte, les informations sont divisées selon la géographie du pays dans Je découvre le Canada (2002). Toutefois, les nations sont présentées comme appartenant à une province ou à un territoire (p. 76). Dans la réalité, une même famille autochtone réside sur des territoires chevauchant les provinces et les territoires. Par exemple, les Algonquins vivent autour de la Baie d'Hudson et leur territoire correspond en réalité au Québec ainsi qu'à l'Ontario. Dans le cas des Micmacs, leur territoire englobe des terres au Québec et dans les Maritimes. Ne s'agit-il pas de divisions géographiques dans une perceptive Eurocentrique? Pourquoi ne pas diviser le contenu selon une conception géographique autochtone ? Cette façon de structurer l'information ne révèle-t-elle pas une vision Eurocentrique de la géographie canadienne?

Finalement, les résultats de l'analyse de la structure et des caractéristiques soulèvent des questionnements quant aux références au passé, quant aux choix de vocabulaire associé à la possession et quant aux tendances vers la dépersonnalisation et vers l'eurocentrisme.

\section{Construction des personnages}

Les façons dont sont construits les personnages soulèvent d'autres questionnements touchant à la reconnaissance des Autochtones. Par exemple, semblant vouloir créer une unité, un manuel met l'emphase sur le contexte immigrant canadien. « $\mathrm{Au}$ Canada, presque tous les gens sont des nouvelles venues ou nouveaux venus, ou ils en descendent » (Je découvre le système, 2002, p. 67). Toutefois, dans le même paragraphe, il 
propose l'idée que « les peuples des Premières Nations et les Inuites et Inuits affirment que leurs ancêtres vivaient au Canada depuis plus longtemps que quiconque peut se souvenir » (Ibid.). Selon cet extrait, puisque ce sont eux-mêmes qui « affirment» leur lien ancestral, cette place particulière donnée aux Premières Nations et aux Inuits est-elle reconnue ? De plus, bien que cet extrait cherche à englober tous les citoyens canadiens, il ne fait pourtant aucunement allusion à l'existence des Métis. D'ailleurs, les représentations de ceux-ci dans les manuels tendent vers une certaine simplification et vers des références à des valeurs Eurocentriques. Par exemple, les fondements des revendications métis sont de l'ordre de la possession : «Ils s'inquiétaient cette fois de tous les nouveaux venus qui envahissaient leurs terres. Ils avaient peur de perdre tous leurs biens » (Je découvre les systèmes de gouvernement du Canada, 2002, p. 61). Serait-ce une projection de valeurs qui ne sont pas celles de Métis? Ne serait-il pas plus pertinent de mentionner la peur de perdre leur culture, leur langue, leur reconnaissance ou leurs droits?

En ce qui concerne le rôle des Autochtones dans l'histoire du Canada, Je découvre les Premières Nations et les explorateurs (2002) présente 41 explorateurs. De ce nombre, seulement six noms autochtones sont précisés : Domagaya et Taignoagny (p. 21), Attickasish, guide de Henday (p. 35), Thanadelthur, une femme ayant contribué au maintien de la paix (p. 43), Louis Riel (p. 45), chef métis, Matonabbee, guide de Hearne (p. 46). Bien qu'il y ait mention des noms, seuls Louis Riel et Thanadelthur sont des personnages qui sont présentés. Les noms des autres sont intercalés entre des contenus dressant le portrait d'autres explorateurs. En résumé, les représentants des Autochtones dans ce manuel portant sur les explorateurs sont moins nombreux et jouent, pour la plupart, un rôle secondaire, un rôle d'aidant. Ces choix ne réduisent-ils pas les contributions autochtones? Les Autochtones ont-ils joué un rôle de secondaire dans l'histoire de l'Amérique?

Puis, quant aux représentations des Autochtones en lien avec des aspects géographiques, il y a matière à réflexion. Entre autres, une citation concernant le droit de vote retient l'attention : «Les Inuits du nord du Canada n'ont obtenu le droit de vote qu'en $1950 »$ (Question de choix, p. 9). Cette spécification de la situation géographique pose question. Pourquoi l'extrait suivant spécifie-t-il la situation géographique ? La situation géographique de ce groupe change-t-elle quelque chose ? Est-ce que cela signifie qu'un Inuit vivant au Sud avait le droit de vote?

Sur cette même lancée, dans le manuel Exploration (1999), il y a aussi présentation de deux cartes : l'une d'entre elles représente des côtes de l'Europe occidentale et de l'Afrique du Nord alors que l'autre se concentre sur une section des Grands Lacs. 

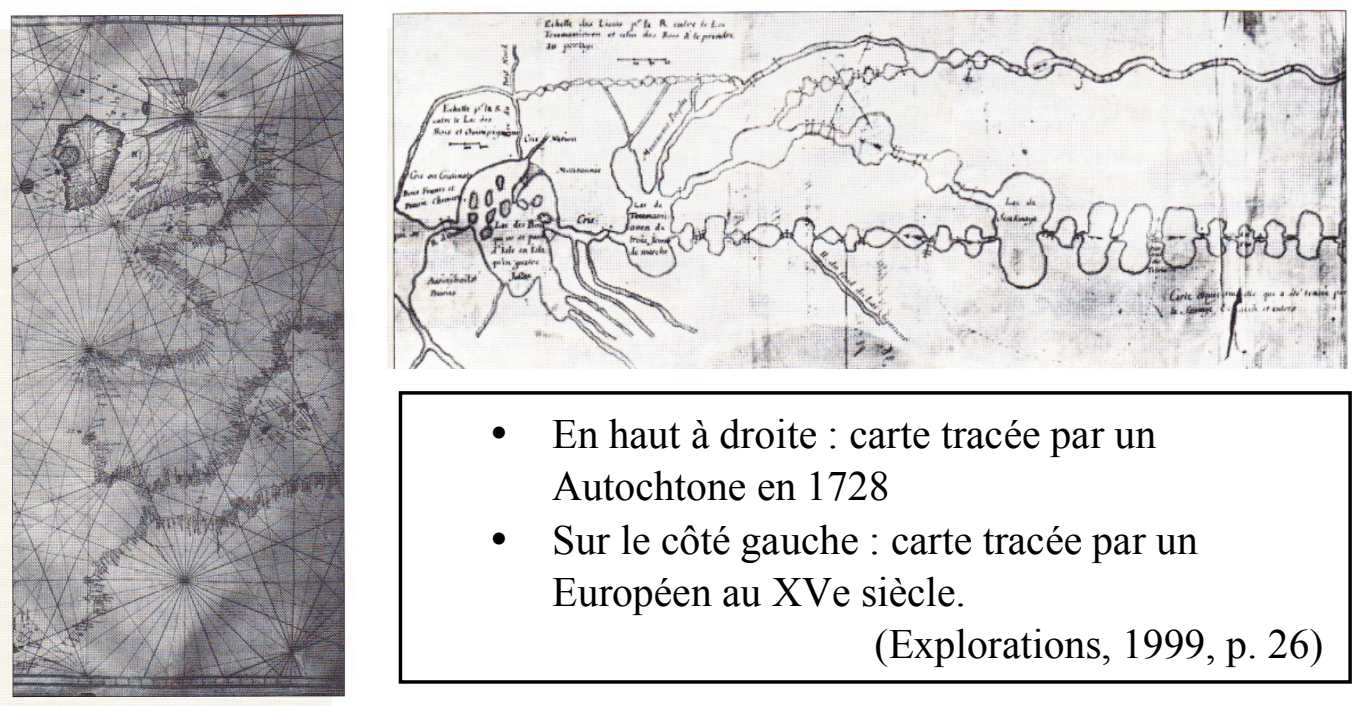

- En haut à droite : carte tracée par un Autochtone en 1728

- Sur le côté gauche : carte tracée par un Européen au XVe siècle.

(Explorations, 1999, p. 26)

Ces deux cartes ne sont pas équivalentes puisqu'elles ne représentent ni le même espace géographique ni la même époque. La carte européenne traversée de lignes laisse croire que l'Européen a eu recours à des calculs précis, idéologie appuyée par l'utilisation de l'adjectif de précision qui ne s'applique qu'aux cartes européennes dans le discours portant sur les avancées de la cartographie en Amérique :

Les Autochtones dessinaient des cartes de leurs territoires de chasse. Ils indiquaient les meilleurs itinéraires aux autres nations amérindiennes ou aux explorateurs européens. À leur tour, les explorateurs européens ont dessiné des cartes précises pour montrer le chemin aux autres. C'était aussi une façon de revendiquer les territoires explorés au nom de leur pays. (ibid.)

Bref, les résultats de l'analyse portant sur la construction des personnages poussent à réfléchir sur la reconnaissance des Autochtones comme peuple originel, sur la reconnaissance de la situation métisse et sur le rôle attribué aux Autochtones quant à leur apport historique.

\section{Omission}

L'omission des Autochtones est fréquente. Dans plusieurs extraits, l'accent est mis sur les deux peuples fondateurs par rapport aux «Autres » : « [p] rès du tiers des Canadiens et des Canadiennes se déclarent d'une origine autre que française et anglaise » (Identité canadienne franco-ontarienne, 2000, p. 23). D'ailleurs, dans Identité canadienne francoontarienne, un extrait mentionne que

la majorité des résidants de la Colombie-Britannique sont d'origine britannique, mais la population est aussi composée de personnes immigrantes 
et descendantes d'immigrants de toutes nationalités dont une forte proportion est asiatique. (p. 5)

Pourquoi omettre de mentionner la présence des Autochtones en Colombie-Britannique ? Aussi, d'autres omissions relèvent du contenu portant sur des personnalités modèles dans La francophonie dans le monde (2000). Effectivement, l'objectif étant de « décrire la contribution de Francophones du Canada et d'ailleurs sur la scène internationale » (p. 55), plusieurs portraits sont présentés. Pourtant, aucun n'est fait d'un représentant francophone du peuple autochtone. Pourquoi ces personnalités plutôt que d'autres incluant les Autochtones?

Dans Je découvre le système de gouvernement au Canada (2002), l'origine des mots tels que Métis, Indiens, et Esquimaux est expliquée.

Auparavant, on appelait les Inuites et les Inuits des « Esquimaux ». Ce mot signifie « personne qui mange de la viande crue ». C'était un terme utilisé par d'autres groupes autochtones, mais aujourd'hui, on préfère utiliser le mot « inuit ». Les Inuites et les Inuits vivent à l'extrême nord du pays. (p. 57)

Ces définitions aident les lecteurs à différencier les groupes autochtones. Par contre, les motifs du changement de termes ainsi que les explications quant aux connotations négatives sont omis. De plus, les omissions quant aux aspects négatifs de l'arrivée des Européens se répètent lorsque, par exemple, seulement quelques inconvénients sont recensés. Pourquoi mettre de l'avant certains éléments de partage et en laisser d'autres comme les maladies, les guerres, les armes, l'alcool et la délocalisation de côté ? Les impacts négatifs qu'ont eus les nouveaux arrivants européens sur les populations sont minimisés aux raisons scientifiques. Qu'en est-il des raisons comportementales?

Finalement, les communautés autochtones sont fréquemment omises selon certains sujets. Les représentations sont inégales, particulièrement dans le cas des Métis. De plus, les impacts négatifs relevant de l'arrivée des Européens sont absents.

\section{Discussion : Quels droits sont non respectés ? Les représentations des Autochtones et le respect des droits}

Les représentations autochtones s'inscrivent-elles dans le respect de l'inculcation des droits et des principes consacrés dans la Convention relative aux droits de l'enfant? Selon les résultats, le discours portant sur les droits territoriaux et la discrimination sont à questionner. D'abord, une première remarque tient compte de la question des droits 
territoriaux puisque l'auteur n'utilise jamais de verbes exprimant la possession telle «avoir ». Dans l'extrait, les Autochtones « habitent» le territoire. Ici, il y a possibilité de déduction quant au malaise entourant les revendications territoriales. Il s'agit probablement d'un terrain glissant et les acteurs derrière la conception et l'approbation de ces manuels, consciemment ou non, ont peut-être préféré ne pas mettre l'accent sur ce débat actuel, sur cette injustice qui ne fait pas bonne presse dans la liste des idéaux canadiens.

Une seconde remarque est faite quant à l'irrespect, l'imposition et la nonconsidération puisque ces notions ne sont pas explorées. Comme le suggère Montgomery (2005ab), l'incompréhension justifie les injustices vécues et la dépersonnalisation engendre la déresponsabilisation des personnes ayant causé les injustices. Il y a peut-être tentative d'atténuer la source et la portée des actes ayant mené aux débats actuels sur les droits des Autochtones. Aussi, comme Tupper (2002) qui déplore que les préjugés entourant les Canadiens d'origine japonaise soient traités au passé et ne révèlent pas le portrait de la situation actuelle, la récurrence des références au passé dans l'échantillon analysé ne permet pas de saisir les situations actuelles des Métis, des Premières Nations ainsi que des Inuits et ne rend pas justice à la réalité vécue.

Concernant l'inculcation du respect des droits, après ces références au passé, les textes étudiés expliquent qu'aujourd'hui, il y a des lois pour protéger les citoyens de tels actes discriminatoires. Le discours propose qu'il y ait des politiques et des règles qui assurent une protection. Alors que les manuels ne traitent pas de l'application de la loi, ce discours donne une image complaisante sur papier et diffuse l'idée d'un refuge en cas d'injustice. En réalité, la législation ne change pas automatiquement l'attitude de tous les citoyens. Ces exemples simplistes ne prennent pas en compte les oppositions, les luttes et les actions prises par les membres du groupe discriminé. Comme Tupper (2002) qui souligne que les luttes des immigrants ne sont pas décrites, l'analyse a démontré que ni le visage actuel, ni les revendications, ni les luttes autochtones ne sont abordés. Face à la discrimination, il y a omission de toutes les tentatives de résistance. De même que Dennick -Brecht (1993) qui critique le fait que les minorités sont souvent victimisées, cette recherche remarque que les Autochtones le sont souvent aussi. D'ailleurs, dans son analyse portant sur les représentations autochtones, l'attribution de ce rôle a aussi été remarquée par Trudel (2000).

L'article 29 de la Convention relative aux droits de l'enfant tend à « favoriser l'épanouissement de la personnalité de l'enfant et le développement de ses dons et de ses aptitudes mentales et physiques, dans toute la mesure de leurs potentialités ». «Inculquer à l'enfant le respect des droits de l'homme et des libertés fondamentales, et des principes consacrés dans la Charte des Nations Unies » et « inculquer à l'enfant le respect (...) de son identité, de sa langue et de ses valeurs culturelles, ainsi que le respect des valeurs nationales du pays dans lequel il vit (...)» (ibid.) sont deux aspects de ce droit. 
L'inculcation de ce respect des droits relève une contradiction. Cette contradiction est le résultat de l'équation de deux aspects : le fait qu'aujourd'hui, il y a des lois pour protéger les citoyens des actes discriminatoires et le contexte de ces discriminations incessamment associées au passé. Il en résulte que le portrait des discriminations actuelles n'est pas proposé aux élèves. Conséquemment, qu'il s'agisse des droits territoriaux ou des injustices, le vécu d'un élève autochtone ne cadre pas avec les représentations de sa communauté dans les manuels scolaires. Finalement, cette contradiction pose problème en ce qui a trait au respect de l'identité, des droits et des valeurs autochtones. Ainsi, si la diversité culturelle est un facteur de développement, la promotion de représentations respectant les valeurs autochtones et l'ouverture à toutes les potentialités seraient bénéfiques au respect des droits des enfants autochtones. En d'autres mots, d'un point de vue légal, afin de reconnaitre l'Autre et de respecter les droits des enfants autochtones, il est impératif d'avoir des manuels plus représentatifs de cette population scolaire.

\section{Les représentations des Autochtones et l'identité}

Les représentations autochtones cadrent-elles avec le droit à une éducation qui respecte pleinement leur identité culturelle ? Sous un regard critique, l'analyse de discours du contenu latent révèle que les représentations sont Eurocentriques et que l'identité est simplifiée. En effet, cette première remarque touche, par exemple, à la division des familles autochtones présentées selon les frontières provinciales. Cette division des groupes autochtones cadre avec une idéologie géographique occidentale et rappelle la dominance Eurocentrique que critiquent Dennick-Brecht (1993) et Hughes (2007) dans les représentations de l' " Autre ». Toujours dans le domaine de la géographie, la comparaison entre une carte autochtone et européenne met en évidence un biais quant à la représentation des compétences cartographiques. Cette comparaison visuelle n'est pas représentative des connaissances géographiques des membres de Premières Nations. En plus de la divergence entre les territoires dessinés, soit une partie du continent européen et un chemin entre des lacs de l'Ontario, le texte suggère que les Européens auraient aidé les Autochtones à développer la cartographie. Pourquoi parler de développement sans considérer le fait que ces différences cartographiques s'expliquent peut-être par une divergence entre les références européennes et autochtones ? Pourquoi ne pas expliquer aux élèves que les connaissances géographiques relevaient de la tradition orale chez les Autochtones? N'était-ce pas plutôt un accommodement fait pour les Européens? Encore une fois, cette formulation qui donne le rôle de connaisseurs aux Européens réfère-t-elle à une forme d'eurocentrisme scientifique ? Sanchez (2007) dénonce aussi ce survol des valeurs et des contributions autochtones dans les manuels scolaires. Ce dernier souligne que les Autochtones sont souvent représentés comme étant nomades, sans technologies et sans terres ou droits d'occupation. 
Une deuxième remarque relève de l'évitement de la complexité et des conflits. Effectivement, comme McCarthy (1990) le mentionne, le contenu décrivant l' "Autre » est traité de façon quelque peu superficielle. Dans le cas des représentations autochtones au sein de l'échantillon, et comme le note aussi Fleming (2006), l'analyse ne révèle pas de descriptions stéréotypées. De plus, cette recherche révèle qu'à l'exception des notions touchant à la technologie, les valeurs entourant les communautés ne se retrouvent pas dans le discours portant sur les Autochtones. Ce constat rejoint les propos de Fleming et Sanchez (2007).

Une troisième remarque porte sur l'inégalité des représentations entre les groupes autochtones : les membres des Premières Nations et les Inuits ont assurément une place particulière qui est non partagée par les Métis. De plus, la nature de ces représentations est aussi controversée : ce n'est pas parce qu'une personne est nommée qu'il est nécessairement question de sa communauté. D'ailleurs, Louis Riel est présenté à quelques reprises de façon sommaire, mais sans soulever le débat relatif aux Métis. Dans cette même veine d'idées, il y a trop souvent présentation d'une personne pour représenter un groupe. Ainsi, cette façon d'inclure l' "Autre » laisse le groupe sous silence (McCarthy, 1990). En d'autres mots, la tendance à porter l'attention sur un événement, ou sur une personne qui a été en tête d'une rébellion, évite que les notions relatives à la culture ne soient abordées.

Bref, à la lumière de ces résultats qui dénoncent les représentations identitaires simplifiées et Eurocentriques, il est pertinent de se questionner par rapport à l'article 28 qui préconise de prendre « des mesures pour encourager la régularité de la fréquentation scolaire et la réduction des taux d'abandon scolaire ». Considérant que le matériel éducatif représentant les diversités culturelles a un impact positif sur les résultats scolaires et sur l'estime de soi des élèves de ces communautés (Diamond et Moore, 1995), y retrouver des représentations plus justes de l'identité autochtone s'inscrirait dans le respect de ce droit.

\section{Conclusion}

Pour conclure, tandis que le projet de loi 13 amène aux documents ministériels une vision interculturelle telle que définie par Abdallah-Pretcelle (1996), les manuels scolaires analysés relèveraient plutôt de l'approche multiculturaliste telle que proposée par Kymlicka (2003). Pour faire suite à l'analyse critique du discours des manuels scolaires, plusieurs remarques sont formulées quant aux représentations des Autochtones dans le contenu latent de ces manuels. Afin que les droits des enfants autochtones soient respectés, il serait important que ces représentations soient plus justes. Cette étude inspire quelques recommandations adressées tant au ministère qu'aux maisons d'édition ou qu'aux auteurs.

D'abord, du côté du ministère de l'Éducation de l'Ontario, les résultats de cette recherche peuvent amener un approfondissement des critères d'évaluation du CLÉ afin 
d'apporter un éclairage quant à l'approbation des manuels recommandés par le ministère sur la liste Trillium. Premièrement, les notions interculturelles devraient être proportionnées selon les sujets et non par rapport à un manuel dans sa globalité. Les références à l' «Autre» ne seraient ainsi plus présentées que dans une section traitant, par exemple, seulement de l'alimentation. Deuxièmement, le curriculum pourrait représenter des Autochtones ayant joué des rôles principaux dans l'histoire. Puis, en ce qui concerne les droits territoriaux, il serait bénéfique d'ajouter des cartes territoriales des dernières décennies afin d'illustrer les pertes et de mieux saisir la nature de ces revendications telles que le propose Bouchard (1999).

Ensuite, les éditeurs et les auteurs devraient utiliser des termes qui confirment les droits d'occupation autochtone et prêter attention aux descriptions de conflits et d'injustices afin que ceux-ci ne soient pas systématiquement justifiés par l'incompréhension, par la dépersonnalisation ou par le passé. Dans la même veine d'idées, un portrait plus actuel de la situation des Autochtones serait requis. Lorsqu'il est question de technologie, il faudrait éviter l'eurocentrisme. Puis, l'inclusion des Métis dans le contenu se rapportant aux Inuits et aux Premières Nations s'avère un impératif.

Finalement, peut-être que l'implication de membres de communautés autochtones dans l'analyse des manuels scolaires permettrait l'inclusion d'une vision du monde qui favoriserait une meilleure représentativité ? Certes, recueillir les commentaires et propositions des élèves autochtones qui ont utilisé ces manuels aiderait à la création de matériel scolaire incluant une représentation plus juste des communautés autochtones en contexte canadien.

À la suite de cette analyse, d'autres questions émergent. Comme l'écrivent Hirsch, Mc Andrew et Couture (2010),

nous sommes passés du manuel « récit», où l'on dit quoi penser, au manuel « outil », où les questions demeurent plus ouvertes, mais aussi plus ambigües et avec plus de latitude laissée aux enseignants. (p. 3)

Approfondir davantage certaines informations relatives à l'exploitation pédagogique et didactique des manuels en classe et traiter de la perception qu'ont élèves et enseignants du discours proposé dans les manuels scolaires complémenteraient cette étude. D'ailleurs, l'échantillon de cette recherche ne couvre pas les notions des guides de l'enseignant.

Malgré ses limites, cette étude n'est qu'un morceau dans ce casse-tête que peuvent être les représentations des Autochtones dans le milieu scolaire. 


\section{Notes}

${ }^{1}$ Je découvre les premières nations et les explorateurs (2002), Je découvre le Canada (2002), Collection mosaïque [5e année]. Question de choix (1999), Collection mosaïque [6e année]. Explorations (1999), Collection mosaïque [6e année]. Laisser sa marque (1999), Collection mosaïque [5e année] : Identité canadienne et franco-ontarienne (2000), Collection mosaïque [6e année] : La Francophonie dans le monde (2000), Je découvre les partenaires commerciaux du Canada (2002).

\section{Remerciements}

L'auteur remercie les deux évaluateurs anonymes pour leurs judicieux conseils.

\section{Références}

Abdallah-Pretceille, M. (1996). Vers une pédagogie interculturelle. Paris : Anthropos.

Assemblée législative de l'Ontario. (2012). Projet de loi 13. Récupéré le 13 avril 2016 à du site de l'Assemblée législative de l'Ontario:

http://ontla.on.ca/web/bills/bills_detail.do?locale=fr\&BillID $=2549$

Bardin, L. (1989). L’Analyse de contenu. $5^{\mathrm{e}}$ édition. Paris : Presses universitaires de France.

Blondin, D. (1990). L'apprentissage du racisme dans les manuels scolaires. Ottawa : Les éditions Agence D’ARC Inc.

Bouchard, G. (1999). La nation québécoise au futur et au passé. Montréal : VLB éditeur, coll. Balises, 87.

CLÉ (Centre canadien de leadership en évaluation). Récupéré le 13 avril 2016 du site de l'organisme : http://www.lecle.com/

Convention relative aux droits de l'enfant. (s. d.). [Droits de l'homme, Haut-Commissariat]. Récupéré le 18 avril 2016 du site de l'organisme: http://www.ohchr.org/fr/professionalinterest/pages/crc.aspx 
Crawford, K. (2004). Inter-Cultural Education: the role of school textbook analysis in shaping a critical discourse on nation and society. Pacific Circle Consortium, $27^{\text {th }}$ annual Conference, Hong Kong Institute of Education. 28.

Dennick-Brecht, M. K. (1993). Developing a more inclusive sociology curriculum: Racial and ethnic group coverage in thirty introductory textbooks. Teaching Sociology, 21(2), 166171. https://doi.org/10.2307/1318638

Diamond, B.J., \& Moore, M.A., (1995). Multicultural literacy: Mirroring the reality of the classroom. Longman Publishers USA.

English Learning Area, Critical literacy. Récupéré le 15 septembre 2009 du site de l'organisme: http://wwwfp.education.tas.gov.au/english/critlit.htm\#whatis

Fleming, W. C. (2006). Getting past our myths and stereotypes about Native Americans. Education Digest: Essential Readings Condensed for Quick Review, 72(7), 51-57.

Gagnon, D. (2006). La nation métisse, les autres métis et le métissage. Les paradoxes de la contingence identitaire. Anthropologie et société. 30 (1), 180-186.

Ghosh, R. (2008). Racism: A hidden curriculum. Education Canada, 48 (4), 26-29.

Gouvernement de l'Ontario (2013, octobre). Effectifs des écoles publiques de l'Ontario. Récupéré le 18 avril 2016 du site du gourvenement : https://www.ontario.ca/fr/donnees/effectifs-des-ecoles-publiques-de-lontario

Hirsch, Mc Andrew et Couture (2010). Quelle est l'image de la diversité culturelle dans le matériel didactique? Récupéré le 13 avril 2016 du site de l'Université de Montréal: http://www.nouvelles.umontreal.ca/recherche/sciences-humaines-lettres/quelle-estlimage-de-la-diversite-culturelle-dans-le-materiel-didactique.html

Hughes, R. L. (2007). A hint of whiteness: History textbooks and social construction of race in the wake of the sixties. Social Studies, 98(5), 201-208.

Largenton, É. (2016, mars). L’Australie a-t-elle été « envahie» ou « découverte»? |ABC Radio Australia. Consulté 20 avril 2016, à l'adresse http://www.radioaustralia.net.au/french/2016-03-31/laustralie-atelle$\% \mathrm{C} 3 \% \mathrm{~A} 9 \mathrm{t} \% \mathrm{C} 3 \% \mathrm{~A} 9-\% \mathrm{C} 2 \% \mathrm{AB}-$ envahie-\%C2\%BB-ou- $\% \mathrm{C} 2 \% \mathrm{AB}-\mathrm{d} \% \mathrm{C} 3 \% \mathrm{~A} 9$ couverte$\% \mathrm{C} 2 \% \mathrm{BB} / 1564640$ 
Loewen, J. (1995). Lies my teacher told me: Everything your American history textbook got wrong. New York: Simon and Schuster.

Kymlicka, W. (2003). La voie canadienne. Repenser le multiculturalisme. Montréal : Éditions Boréal.

McAndrew, M. (1987). Le traitement de la diversité raciale, ethnique et culturelle dans le matériel didactique au Québec. Montréal : CCCI.

Mc Andrew, M. (2006). Éducation à la citoyenneté et éducation interculturelle : le cas québécois. Dans Y. Lenoir et C. Xypas (dir.), Éducation à la citoyenneté et diversité culturelle. Paris : PUF.

McCabe, P. (1996). A case grammar analysis of representation of African-Americans in current fifth grade social studies textbooks. Reading Horizons, 36, 380-401.

McCarthy, C. (1990). Multicultural education, minority identities, textbooks, and the challenge of curriculum reform. Journal of Education, 172(2), 118-129.

McCarthy, C. (1990). Race and education in the United States: The multicultural solution. Interchange, 21(3), 45-55.

McCarthy, C. (1991). Multicultural approaches to racial inequality in the United States. Oxford Review of Education, 17(3), 301-316.

McGregor, S. (2003). Critical science approach-a primer. Récupéré le 13 avril 2016 du site: http://www.kon.org/cfp/critical_science_primer.pdf_2009.

Ministère de l'Éducation de l'Ontario. (2004). Politique d'aménagement linguistique de l'Ontario. Toronto, le ministère.

Ministère de l'Éducation de l'Ontario. (2008). Lignes directrices sur l'approbation des manuels scolaires. Récupéré le 13 avril 2016 du site du ministère :

http://www.tfo.org/listetrillium/pdf/Textbook_Guide_Fr_2008\%20Revised.pdf

Ministère de l'Éducation de l'Ontario (2009). Une approche culturelle de l'enseignement pour l'appropriation de la culture dans les écoles de langue française de l'Ontario : Cadre d'orientation et d'intervention. Récupéré le 13 avril 2016 du site du ministère: https://www.edu.gov.on.ca/fre/amenagement/PourLaFrancophonie2009.pdf 
Ministère de l'Éducation de l'Ontario. (2013). Curriculum de l'Ontario. Études sociales de la $1^{\text {re }}$ à la $6^{\mathrm{e}}$ année. Récupéré le 13 avril 2016 du site du ministère : http://www.tfo.org/listetrillium/pdf/Textbook_Guide Fr_2008\%20Revised.pdf

Moirand, V. (2014, juin). Libérer le potentiel des élèves autochtones ontariens | Canadian Education Association (CEA). Récupéré le 18 avril 2016 du site Canadian Education Association (CEA): http://www.cea-ace.ca/education-canada/article/lib\%C3\%A9rerle-potentiel-des-\%C3\%A91\%C3\%A8ves-autochtones-ontariens

Montgomery, K. (2005a). Banal race thinking: Ties of blood, Canadian history textbooks and ethnic nationalism. Pedagogical Historica: International Journal of the History of Education, 41(3), 313-336.

Montgomery, K. (2005b). Imagining the Antiracist State: Representations of racism in Canadian history textbooks. Discourse: Studies in the cultural politics of education, 26 (4), 427442 .

Moreau, J. (2003). Schoolbook nation: Conflicts over American history textbooks from the Civil War to the present. Ann Arbor: University of Michigan Press.

Nasser, R., \& Nasser, I. (2008). Textbooks as a vehicle for segregation and domination: State efforts to shape Palestinian Israelis' identities as citizens. Journal of Curriculum Studies, 40(5), 627-650.

Peck, C. L., Sears, A., \& Donaldson, S. (2008). Unreached and unreasonable: Curriculum standards and children's understanding of ethnic diversity in canada. Curriculum Inquiry, $38(1), 63-92$.

Razai-Rashti, G. M., \& McCarthy, C. (2008). Race, text, and the politics of official knowledge: A critical investigation of a social science textbook in Ontario. Discourse: Studies in the Cultural Politics of Education, 29(4), 527-540.

Sanchez, T. R. (2007). The depiction of Native Americans in recent (1991-2004) secondary American history textbooks: How far have we come? Equity \& Excellence in Education, 40(4), 311-320.

Sleeter, C.E. \& Grant, C.A" (1991). Race, class gender, and disability in textbooks. In M.W. Apple \& L. Christian-Smith (Eds.), Politics and the textbook (pp. 78-110). New York: Routledge, Chapman, \& Hall. 
Statistique Canada (2006). Recensement de 2006 : Peuples autochtones. (s. d.). Récupéré le 18 avril $2016 \mathrm{du}$ site de l'organisme : http://www12.statcan.gc.ca/censusrecensement/2006/rt-td/ap-pa-fra.cfm

$\mathrm{Su}, \mathrm{Y}$. (2007). Ideological representations of Taiwan's history: An analysis of elementary social studies textbooks, 1978-1995. Curriculum Inquiry, 37(3), 205-237.

Trudel, P. (2000). Histoire, neutralité et Autochtones : une longue histoire... Revue d'histoire de l'Amérique française, 53(4), 528-540.

Tupper, J. (2002). Silent voices, silent stories: The representation of Japanese Canadians in social studies textbooks. Alberta. Journal of Educational Research, 48(4), pp. 327-340.

Vincent, S. et Arcand, B. (1979). L'image de l'Amérindien dans les manuels scolaires du Québec: ou, Comment les Québécois ne sont pas des sauvages. Ville LaSalle, Québec: Éditions Hurtubise.

Zimmerman, J. (2002). Whose America? Culture wars in the public schools. Cambridge, MA: Harvard University Press.

\section{Liste des manuels analysés :}

Francis, D. (2002). Je découvre les premières nations et les explorateurs. Montréal: Éditions de la Chenelière.

Francis, D. (2002). Je découvre le système de gouvernement au Canada. Montréal: Éditions de la Chenelière.

Gutsole, M. (2002). Je découvre le Canada. Montréal: Éditions de la Chenelière.

Peturson, R. (1999). Collection mosaïque [5e année]. Question de choix : [fascicule de l'élève. Montréal: Éditions de la Chenelière.

Peturson, R. (1999). Collection mosaïque [6e année]. Explorations : [fascicule de l'élève. Montréal: Éditions de la Chenelière.

Peturson, R. (1999). Collection mosaïque [6e année]. Laisser sa marque : [fascicule de l'élève. Montréal: Éditions de la Chenelière. 
Renaud-Charette, C. (2000). Collection mosaïque [5e année]. Identité canadienne et francoontarienne: [fascicule de l'élève. Montréal: Éditions de la Chenelière.

Renaud-Charette, C. (2000). Collection mosaïque [6e année]. La Francophonie dans le monde : [fascicule de l'élève. Montréal: Éditions de la Chenelière.

Ursel, E. (2002). Je découvre les partenaires commerciaux du Canada. Montréal: Éditions de la Chenelière 